\title{
Characterization of Oxide Layers on Stainless Steel Using Auger Electron Spectroscopy (AES), Scanning Electron Microscopy-Focused Ion Beam (SEM-FIB) and Transmission Electron Microscopy (TEM)
}

Henry Ajo

SRNL, Aiken, South Carolina, United States

The protective oxide layer on stainless steels accounts for their ability to weather environments that would corrode other steels (Adams, 1983). Due to the nature of this oxide layer, Auger Electron Spectroscopy (AES) has been used to characterize the elemental composition of this complex oxide (Ajo et al, 2014.). New sample preparation and characterization methods are explored to determine the information that can be determined using newer Scanning Electron Microscopy- Focused Ion Beam (SEM-FIB) and Transmission Electron Microscopy (TEM).

AES provides a surface sensitive spectroscopy limited to the outermost 5-10 nm of the surface. Depth profiling is performed to excavate the surface and determine elemental composition as a function of sputtering time. Using previous calibrations, the sputtering time is converted to a depth in a known material. In this way, by sputtering and taking AES measurements at intervals, the composition of the material is learned as a function of depth.

Using AES depth profiles, oxide layers on stainless steel generally follow the same pattern: a rise in oxygen signal to a maximum, followed by a steady decrease to a stable value, generally zero, if the surface begins relatively clean. The depth profile is carried out deep enough to result in an oxygen free surface which shows a plateauing of the values of the other constituents of the stainless steel to show that the oxide layer has been removed and that the measurement has proceeded beyond the oxide layer into the bulk.

SEM-FIB will be used as a sample preparation technique for TEM lamella. The SEM-FIB can be used to locate a region of interest for lamella preparation and can in fact be used to take some measurements of the oxide layer, but generally for thinner layers, the imaging resolution of the SEM-FIB will not be sufficient and a lamella will need to be prepared for examination in the TEM. In the TEM, high spatial resolution imaging of the oxide layer can be performed to determine its dimensions.

References

Adams, R.O. (1983). A Review of the Stainless-Steel Surface. J. Vac. Sci. Technol. A 1, 12-18.

Ajo, Henry \& Blankenship, Donnie \& Clark, Elliot. (2014). Analysis of Passivated A-286 Stainless Steel Surfaces for Mass Spectrometer Inlet Systems by Auger Electron and X-ray Photoelectron Spectroscopy and Scanning Electron Microscopy. Metallogr., Microstruct., Anal.3, 263-271. 